\title{
Angina-like chest pain: a joint medical and psychiatric investigation
}

\author{
S.M. Colgan, ${ }^{1}$ P.M. Schofield, ${ }^{2}$ P.J. Whorwell, ${ }^{1}$ D.H. Bennett,${ }^{2}$ N.H. Brooks ${ }^{2}$ \\ and P.E. Jones ${ }^{2}$
}

${ }^{1}$ Department of Medicine, University Hospital of South Manchester, Manchester M20 8LR and

${ }^{2}$ Department of Cardiology, Wythenshawe Hospital, Manchester, UK.

\begin{abstract}
Summary: Sixty three patients with chest pain typical of angina and who had normal coronary angiograms were investigated for left ventricular, oesophageal and psychiatric abnormalities. An additional 21 patients, age and sex matched, who had angina and significant coronary artery disease were also studied.

Eighty six per cent of the 63 patients without evidence of coronary artery disease could be demonstrated to have a physical abnormality (left ventricular dysfunction in $35 \%$, oesophageal disorder $51 \%$ ). There was, however, a wide variation in the incidence of psychiatric morbidity between the diagnostic subgroups $-\mathbf{1 8 \%}$ in left ventricular dysfunction, $29 \%$ in those with coronary artery disease and $59 \%$ in patients with oesophageal disorders $(P<0.01)$. Thus failure to identify left ventricular dysfunction and inclusion of such subjects in psychological assessments of 'angiogram-negative' chest pain might give misleading results.

This study confirms that patients with angina and normal coronary angiography have a high incidence of oesophageal disorders. However psychiatric illness is also common in this group of subjects and management needs to take both these factors into account.
\end{abstract}

\section{Introduction}

It is not uncommon to find angiographically normal or near normal coronary arteries in patients presenting for investigation of angina-like chest pain. $^{1,2}$ There is now good evidence that a proportion of these patients can be shown to have an oesophageal disorder ${ }^{3}$ or an abnormality of left ventricular function. ${ }^{4}$ There remains, however, a group of patients in whom there is no demonstrable organic pathology. Channer et al. ${ }^{5}$ demonstrated that the probability of a negative exercise test was much greater in those patients with atypical angina and who were anxious and depressed than in those with typical angina but without psychiatric morbidity. Bass et al. ${ }^{6}$ demonstrated an association between psychiatric morbidity, hyperventilation syndrome and the absence of significant coronary artery disease but their patients were not subjected to investigation of the oesophagus. An association between oesophageal contraction abnormalities and psychiatric disorders ${ }^{7}$ has also been reported but the subjects studied had a wide variety of symptomatology and were not routinely submitted for

Correspondence: P.J. Whorwell, M.D., F.R.C.P.

Accepted: 24 March 1988 coronary angiography, oesophageal studies and psychiatric assessment.

It was the purpose of this study to perform a systematic psychiatric assessment on patients attending for extensive investigation of angina-like chest pain which included coronary angiography, assessment of left ventricular function and oesophageal function studies.

\section{Patients and methods}

Sixty three patients undergoing investigation for angina-like chest pain in whom coronary angiography was normal were studied. Initially all patients had quantitative assessment of left ventricular regional wall motion. The percentage of systolic shortening in seven hemiaxes was calculated from the resting left ventricular angiogram. A hypokinetic segment was defined as a hemiaxis where this was less than two standard deviations from the normal mean. ${ }^{4}$ Upper gastrointestinal endoscopy and oesophageal motility measurements ${ }^{8}$ were performed and 52 patients had 24 hour ambulatory $\mathrm{pH}$ monitoring. The age and sex incidence of this

(C) The Fellowship of Postgraduate Medicine, 1988 
group of 63 patients was found to be different from that of an unselected population of patients with coronary artery disease. For comparison, therefore, 21 patients with coronary disease, of a similar age and sex distribution as the angiographically normal subjects, were selected from consecutive patients found to have coronary artery disease at angiography and submitted to oesophageal manometry. Thus it was possible to assign patients to one or more of the following diagnoses: coronary artery disease, left ventricular dysfunction without coronary artery disease, oesophageal motility disorder, oesophageal reflux disease or no demonstrable abnormality.

Each patient was interviewed blind by a psychiatrist using the Clinical Interview Schedule (CIS) ${ }^{9}$ at the time of the oesophageal studies, i.e., after coronary angiography but before being told the results and significance of the investigations. The CIS is a semi-structured interview which rates a number of defined psychiatric symptoms and signs to derive a total score, a severity rating and a diagnosis according to the International Classification of Diseases. ${ }^{10}$ Assessments made by the CIS have been shown to have a high degree of reliability between one psychiatrist and another. ${ }^{9}$

\section{Results}

Of the 84 patients studied 21 had coronary artery disease ( 8 males, 13 females, mean age 50 years) and 63 had normal coronary angiography (23 males, 40 females, mean age 47 years). Of the latter group 22 patients $(35 \%)$ were detected as having left ventricular regional wall motion abnormalities and 36 had an oesophageal disorder demonstrated by $\mathrm{pH}$ monitoring or manometry as we have already reported. ${ }^{8}$ For the purpose of comparison patients were assigned to one of 5 diagnostic categories: (1) Coronary artery disease: 21 patients (CAD) (including 2 with oesophageal motility disorder). (2) Left ventricular dysfunction: 22 patients (LVD) (including 4 with oesophageal motility disorder). (3) Oesophageal motility disorder: 27 patients (OMD) (including 19 subjects with gastrooesophageal reflux). (4) Gastro-oesophageal reflux alone: 5 patients (GOR). (5) No detectable abnormality: 9 patients (NA).

A total of 34 patients were considered to have a psychiatric disorder, 18 had a depressive illness, 13 an anxiety neurosis and 3 had agoraphobia. The distribution of psychiatric illness amongst diagnostic categories is shown in Table I.

The prevalence of psychiatric disorders in those patients with coronary artery disease $(29 \%)$ was less than half that in those with an oesophageal

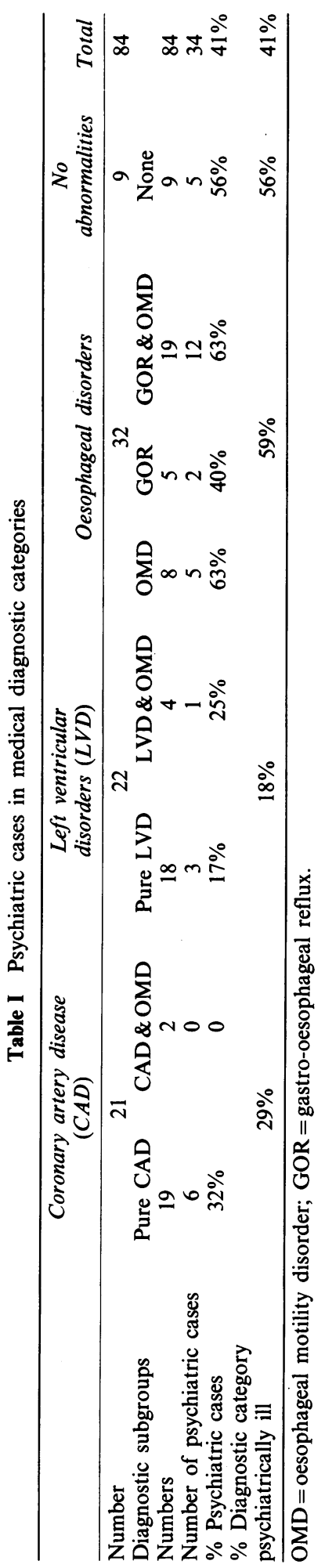


disorder $(59 \%)$ but due to low numbers in each of the diagnostic subgroups this did not achieve statistical significance $\left(\chi^{2}=3.68\right)$. However, there was no significant difference between the prevalence of psychiatric disorders in those patients with coronary artery disease and those with left ventricular abnormalities $(P=0.42)$, thus for comparison these two groups were combined to form one with cardiac abnormalities. The prevalence of psychiatric disorder in this group was significantly less than in those patients with chest pain and an oesophageal disorder, $23 \%$ versus $59 \% \quad(P<0.01)$. In those patients with no detectable abnormality and those with an oesophageal disorder the prevalence of psychiatric disorder was similar and significantly greater than in those patients with a cardiac abnormality $(P=0.001)$. In particular those patients with oesophageal abnormalities had approximately twice the prevalence of psychiatric disorder as did the patients with coronary artery disease and three times that of those with left ventricular dysfunction $(P<0.01)$.

\section{Discussion}

The prevalence of psychiatric morbidity in patients without coronary artery disease $(\mathbf{4 4 . 5 \% )}$ ) is less than that found by Bass et al. $(61 \%)^{6}$ in their study of patients with chest pain and normal coronary arteries. They suggested that the symptoms may be due to the somatic manifestations of anxiety and overbreathing rather than the consequences of underlying cardiac disease, although their study included patients with atypical angina. In this study, when the 63 patients without coronary artery disease but with typical angina were subjected to further cardiac and oesophageal investigations, abnormalities could be detected in $54(86 \%)$. The prevalence of psychiatric disorders was not evenly distributed amongst the various diagnostic categories but was greatest in those with oesophageal abnormalities $(59 \%)$. Thus an association between psychiatric morbidity, oesophageal disorders and absence of cardiac pathology in patients presenting with pain typical of angina has been demonstrated.

Explanations of this association include the possibility that the abnormal oesophageal motility detected is a concomitant of a psychiatric disorder, similar to the abnormal colonic motility found in psychoneurotic patients described by Latimer ${ }^{11}$ in his investigation of the irritable bowel syndrome.

\section{References}

1. Proudfit, W.L., Shirey, E.K. \& Stones, F.M. Selective cine coronary arteriography: correlation with clinical findings in 1000 patients. Circulation 1966, 33: 901-910.
Alternatively, symptoms of an oesophageal disorder may occur frequently in the community but with the majority of sufferers not seeking help for their problem as is seen in subjects with symptoms of bowel dysfunction. ${ }^{12,13}$ In this situation the independent occurrence of a psychiatric disorder may bring the patient to the attention of their doctors at a stage where their symptoms alone would not have done so. Such selective referral would lead to a spuriously high association between psychiatric illness and oesophageal motility disorders in subjects studied in the outpatient department.

A history of chest pain which is typical of angina pectoris, with normal coronary angiograms and with an abnormal exercise electrocardiogram is often referred to as syndrome X. ${ }^{14}$ Some of these patients have been demonstrated to have oesophageal disorders ${ }^{3,8}$ and others abnormal left ventricular function. ${ }^{4}$ Several mechanisms have been proposed in order to explain ischaemia in the absence of coronary occlusive disease. Richardson et al. ${ }^{15}$ suggested the possibility of cardiomyopathy on the basis of myocardial biopsy specimens. More recently it has been shown that some patients have a decreased coronary vasodilator reserve. ${ }^{16}$ This implies a limited capacity to decrease coronary resistance and increase coronary bloodflow in response to increased demand. Our patients with abnormal left ventricular regional wall motion demonstrated features during treadmill exercise testing that were suggestive of myocardial ischaemia. 4,8 Patients with congestive cardiomyopathy often complain of chest pain, despite the presence of normal or even large coronary arteries, and they have been found to have reduced coronary blood flow at rest and during cardiac pacing. ${ }^{17}$

These results confirm the association between chest pain, psychiatric morbidity and negative investigations for coronary disease, but this group is not diagnostically homogeneous. The clinician should be particularly alert for psychiatric illness in those subsequently shown to have oesophageal disorders and in those without demonstrable abnormalities. It remains to be determined whether psychiatric treatment of such patients could relieve their symptoms or even possibly alter motility patterns.

\section{Acknowledgements}

The authors would like to thank Dr C.L. Bray and Dr C. Ward for permission to study patients under their care.

2. Kemp, H.G., Vokonas, P.S., Cohn, P.F. \& Gorlin, R. The angina syndrome associated with normal coronary arteriograms: report of a six year experience. $\mathrm{Am}$ $J$ Med 1973, 54: 735-742. 
3. De Caestecker, J.S., Blackwell, J.N., Brown, J. \& Heading, R.C. The oesophagus as a cause of recurrent chest pain: which patients should be investigated and which tests should be used? Lancet 1985, ii: 1143-1146.

4. Schofield, P.M., Brooks, N.H. \& Bennett, D.H. Left ventricular dysfunction in patients with angina pectoris and normal coronary angiograms. Br Heart $J$ 1986, 56: $327-333$.

5. Channer, K.S., Papouchado, M., James, M.A. \& Rees, J.R. Anxiety and depression in patients with chest pain referred for exercise testing. Lancet 1985, ii: 820-823.

6. Bass, C., Cawley, R.H., Wade, C. et al. Unexplained breathlessness and psychiatric morbidity in patients with normal and abnormal coronary arteries. Lancet 1983 , i: $605-609$.

7. Clouse, R.E. \& Lustman, P.J. Psychiatric illness and contraction abnormalities of the esophagus. $N$ Engl J Med 1983, 309: 1337-1342.

8. Schofield, P.M., Brooks, N.H., Colgan, S. et al. Left ventricular function and oesophageal function in patients with angina pectoris and normal coronary angiograms. Br Heart J 1987, 58: 218-224.

9. Goldberg, D.P., Cooper, B., Eastwood, M.R., Kedward, H.B. \& Shepherd, M. A standardised psychiatric interview for use in community surveys. $\mathrm{Br}$ J Prev Soc Med 1970, 24: 18-23.

10. World Health Organisation, Mental Disorders: Glossary and guide to their classification in accordance with the Ninth Revision of the ICD. WHO, Geneva, 1978.
11. Latimer, P., Sarna, S., Campbell, D., Latimer, M., Waterfall, W. \& Daniel, E.E. Colonic motor and myoelectrical activity: a comparative study of normal subjects, psychoneurotic patients, and patients with irritable syndrome. Gastroenterology 1980, 80: 893-901.

12. Thompson, W.G. \& Heaton, K.W. Functional bowel disorders in apparently healthy people. Gastroenterology 1980, 79: 283-288.

13. Drossman, D.A., Sandler, R.S., McKee, D.C. \& Lovitz, A.J. Bowel patterns among subjects not seeking health care. Gastroenterology 1982, 83: 529-534.

14. Editorial. Syndrome X. Lancet 1987, ii: 1247-1248.

15. Richardson, P.J., Livesley, B. \& Oram, S. Angina pectoris with normal coronary arteries. Transvenous myocardial biopsy in diagnosis. Lancet 1974, ii: 677-680.

16. Opherk, D., Zebe, H., Weihe, E. et al. Reduced coronary dilatory capacity and ultrastructural changes of the myocardium in patients with angina pectoris and normal coronary arteriograms. Circulation 1981, 63: $817-825$.

17. Pasternac, A., Noble, J., Streulens, Y., Elie, R., Henschke, C. \& Bourassa, M.G. Pathophysiology of chest pain in patients with cardiomyopathies and normal coronary arteries. Circulation 1982, 65: 778-789. 